Int. J. Dev. Biol. 59: 443-451 (2015)

doi: $10.1387 / \mathrm{ijdb} .150040 \mathrm{ds}$

\title{
Direct regulation of siamois by VegT is required for axis formation in Xenopus embryo
}

\author{
HONG-YAN LI ${ }^{1,2,3}$, WARIF EL YAKOUBI ${ }^{1,2}$ and DE-LI SHI*,1,2 \\ 'Sorbonne Universités, UPMC Univ Paris 06, Institut de Biologie Paris-Seine (IBPS), UMR 7622, Laboratory of \\ Developmental Biology, Paris, France, ${ }^{2}$ CNRS, IBPS, UMR 7622, Laboratory of Developmental Biology, Paris, France and \\ ${ }^{3}$ Laboratory for Evolution and Development, Department of Marine Biology, Ocean University of China, Qingdao, China
}

\begin{abstract}
The homeobox gene siamois is one of the earliest genes expressed in the Spemann organizer and plays a critical role in the formation of the dorsoventral axis. It is directly regulated by maternal Wnt signalling and functions as an essential zygotic intermediary between maternal factors and the formation of the Spemann organizer. The maternal T domain transcription factor VegT interacts with Wnt signalling and is also involved in the formation of the Spemann organizer. However, the molecular mechanism of this functional interaction is not fully understood. Here we show that VegT is required for siamois expression through direct binding to the T-box binding sites in the siamois promoter. Mutational analysis of each of the five consensus T-box binding sites suggests that the proximal site close to the transcription start site is essential for activation of siamois promoter by VegT, while individual mutation of the four distal sites has no effect. VegT and Wnt signalling also functionally interact and are mutually required for siamois expression. In particular, VegT synergizes with Tcf1, but not Tcf3 and Tcf4, to induce siamois expression, and this is independent of Tcf/Lef-binding sites or the proximal T-box binding site in the siamois promoter. We further extend previous observations by showing that VegT cooperates with maternal Wnt signalling in the formation of the dorsoventral axis. These results demonstrate that maternal VegT directly regulates siamois gene transcription in the formation of the Spemann organizer, and provide further insight into the mechanism underlying the functional interaction between Veg $T$ and Wnt signalling during development.
\end{abstract}

KEY WORDS: Dorsoventral axis, VegT, siamois, Wnt, Xenopus

\section{Introduction}

During early amphibian development mesoderm is formed through inductive signals emanating from endoderm cells of the vegetal hemisphere and acting on the overlying equatorial cells. The molecular nature underlying this fundamental inductive event has been extensively studied and now partly elucidated. An essential pathway for mesoderm formation is triggered by VegT, a $T$ domain family member of transcription factor, also known as Brat, Xombi and Antipodean (Lustig et al., 1996; Stennard et al., 1996; Zhang and King, 1996; Horb and Thomsen, 1997). VegT activates mesoderm formation by regulating the expression of TGF- $\beta$ family genes, including Xnr1, Xnr2, Xnr4 and derriere (Kofron et al., 1999; Xanthos et al., 2001), as well as a panel of other direct target genes (Tada et al., 1998; Casey et al., 1999; Taverner et al., 2005; Sudou et al., 2012). Maternal Wnt signalling also plays an essential role for the establishment of Spemann organizer in the dorsal region by activating the expression of specific target genes, in particular the homeobox gene siamois, which plays a key role in the formation of dorsoventral axis (Lemaire et al., 1995; Fan and Sokol, 1997; Kessler, 1997). Thus, siamois is directly regulated by maternal Wnt signalling and functions as an essential zygotic intermediary between maternal factors and the formation of Spemann organizer (Carnac et al., 1996; Brannon et al., 1997; Fan et al., 1998). It is therefore clear that the formation of dorsoventral axis involves a combinatory action of distinct signalling pathways and transcription factors.

VegT is supplied as a maternal mRNA localized in the vegetal

Abbreviations used in this paper: TCF/LEF, T-cell factor/lymphoid enhancer factor.

*Address correspondence to: De-Li Shi. Sorbonne Universités, UPMC Univ Paris 06, Institut de Biologie Paris-Seine (IBPS), UMR 7622, Laboratory of Developmental Biology, F-75005, Paris, France.Tel: 33-1-4427-2772; Fax: 33-1-4427-3445. E-mail: de-li.shi@ upmc.fr

Supplementary Material (seven figures) for this paper is available at: http://dx.doi.org/10.1387/ijdb.150040ds

Accepted: 14 May 2015.

ISSN: Online 1696-3547, Print 0214-6282

(C) 2015 UBC Press

Printed in Spain 
A

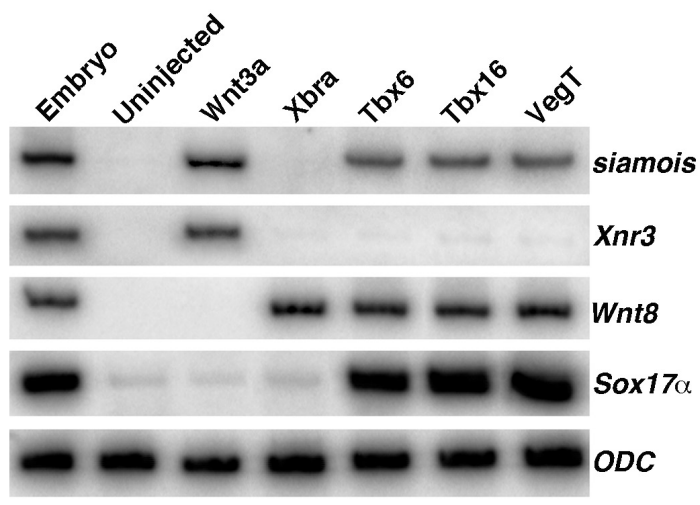

B

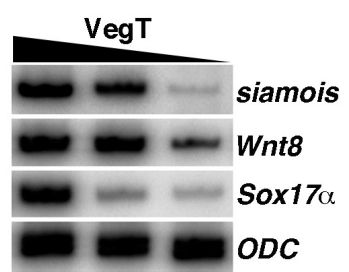

Fig. 1.T domain transcription factors induce ectopic siamois expression in ectoderm cells. (A) Comparison of the siamois inducing activity between Wnt3a and different T domain transcription factors. Embryos at the 2-cell stage were injected with indicated synthetic mRNA in the animal pole region and ectoderm explants were dissected at the early gastrula stage for RT-PCR analysis of gene expression, as indicated. Wnt3a induces the expression of both siamois and Xnr3, but not other mesoderm and endoderm genes. Tbx6, Tbx16 and VegT similarly induce the expression of siamois, Wnt8 and Sox17a genes, but not Xnr3. Xbra only induces the expression ofWnt8. (B) VegTinduces siamois expression in a dose-dependent manner. The expression of siamois is induced by high and intermediate doses of VegT mRNA, while low dose of VegT mRNA only induces Wnt8 expression. The endoderm gene Sox $17 \alpha$ is induced by a high dose of VegT mRNA. ODC was used as a loading control for input RNAs.

hemisphere of oocytes and early embryos, it is also transcribed zygotically within the entire marginal zone of the early gastrula (Heasman, 1997; White and Heasman, 2008). Deleption of maternal VegTtranscripts prevents the formation of mesendoderm (Zhang et al., 1998), while its mis-expression in ectoderm suppresses head formation (Zhang and King, 1996). This suggests that VegT may operate sequentially in several developmental pathways during embryogenesis, including dorsoventral and posterior patterning. Thus, in addition to initiate general mesoderm induction, VegT is also involved in the formation of Spemann organizer and in the patterning of embryonic axis, and its interaction with maternal Wnt signalling in these processes is well documented (Agius et al., 2000; Xanthos et al., 2002). In particular, both VegT and $\beta$-catenin are required for the activation of a panel of mesendoderm genes at the late blastula and early gastrula stages (Agius et al., 2000; Xanthos et al., 2002). Thus, VegT represents an important pathway for the formation and function of Spemann organizer. However, the molecular mechanism underlying this functional interaction is not fully understood.

Here we show that VegT is directly involved in the expression of the Spemann organizer gene siamois through two related mechanisms. First, we demonstrate that it directly activates siamois expression by binding to the T-box binding sites present in the siamois promoter. This induction occurs independently of Wnt signalling. Second, maternal VegT and Wnt signalling functionally interact and are mutually required for siamois expression. In particular, VegT strongly synergizes with Tcf1 to induce siamois expression, even in the absence of Tcf/Lef-binding sites. These results unravel a novel mode of siamois regulation in the formation of Spemann organizer in Xenopus.

\section{Results}

Induction of siamois ectopic expression by a subset of $T$ domain proteins in ectoderm cells

We have previously used a hormone-inducible form of Tbx6VP16, Tbx6VP16-GR, in which Tbx6VP16 was fused with the ligandbinding domain of the human glucocorticoid receptor (Fig. S1A), to identify Tbx6 target genes during Xenopus early development ( $\mathrm{Li}$ et al., 2006). Synthetic Tbx6VP16-GR mRNA (200 pg) was injected in the animal pole region at the 2-cell stage and ectoderm explants were dissected at early gastrula stage and incubated in $10 \mu \mathrm{M}$ dexamethasone (DEX) for 1.5 hours. RT-PCR was performed to analyze the expression of a panel of early mesoderm and endoderm markers. Among different genes whose expression was induced in this condition, we have made an unexpected observation that the expression of siamois, a specific target gene of maternal Wnt signalling (Carnac et al., 1996; Brannon et al., 1997; Fan et al., 1998), was also strongly induced, while no expression of Xnr3, another specific Wnt target gene (McKendry et al., 1997), was detected (Fig. S1B). This indicates that Tbx6VP16 may directly induce siamois expression.

To see if other T domain proteins were also able to induce siamois expression in ectoderm explants, we injected synthetic mRNA corresponding to Xbra (500 pg), Tbx6 (500 pg), zebrafish Tbx16
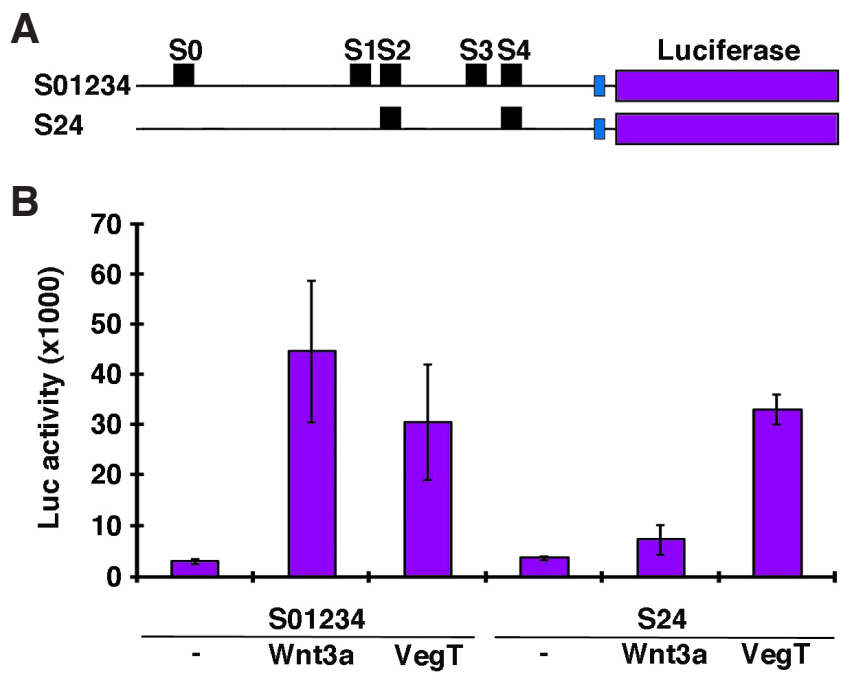

Fig. 2. VegT activates siamois promoter reporter independently ofTcf/ Lef-binding sites. (A) Schematic representation of wild-type (S01234) and mutated (S24) siamois promoter luciferase reporters, with the location of Tcf/Lef sites indicated (not in scale). (B) Both Wnt3a and VegT activate the S01234 siamois promoter with 3 sites (SO, S1, and S3) that conform to the TCf/Lef- consensus binding sites and 2 sites (S2 and S4) that diverge at the 3'-most base. VegT, but not Wnt3a, also activates the $S 24$ siamois promoter mutated at the SO, S1 and S3Tcf/Lef-binding sites. Data represent the mean of triplicate experiments (error bars indicate s.d.). 
A

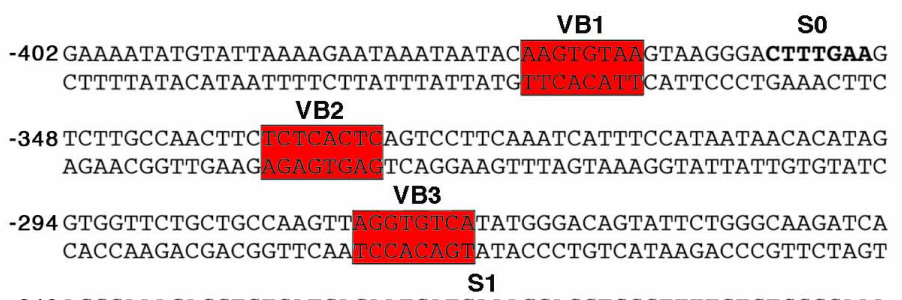

-240 AGGGAAACACGTGTCATCAGAATCATCAAAGGACCTCCCTTTTGTGTCCCCAAA TCCCTTTGTGCACAGTAGTCTTAGTAGTTTCCTGGAGGGAAAACACAGGGGTTT S2

VB4

-186 TCACATCTTTGACCTTGCCAATTCTTTCAGGGTGCATATGTATAAA AGTGTAGAAACTGGAACGGTTAAGAAAGTCCCACGTATACATATTT

53

-132 ACTGACCAATAAACCACGAGCAACAGTACATCAAAGTATTTATATTTTTTTCAT TGACTGGTTATTTGGTGCTCGTTGTCATGTAGTTTCATAAATATAAAAAAAGTA S4

-78 TTCCCCCAAAACACATCCTGGACACACCCTTTGTCCCCTGTTGATATAAAGTCT AAGGGGGTTTTGTGTAGGACCTGTGTGGGAAACAGGGGACAACTATATTTCAGA

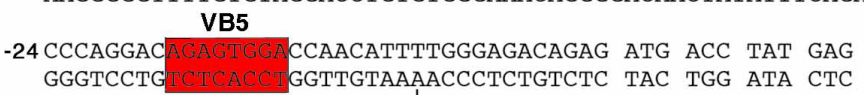
$\stackrel{\text { MACCCTCTGTCTC TAC TGG ATA }}{\longrightarrow}$ M T T

B

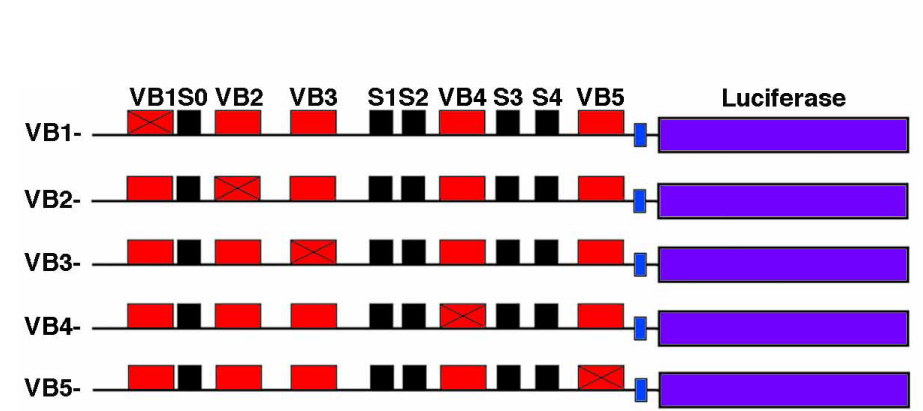

E
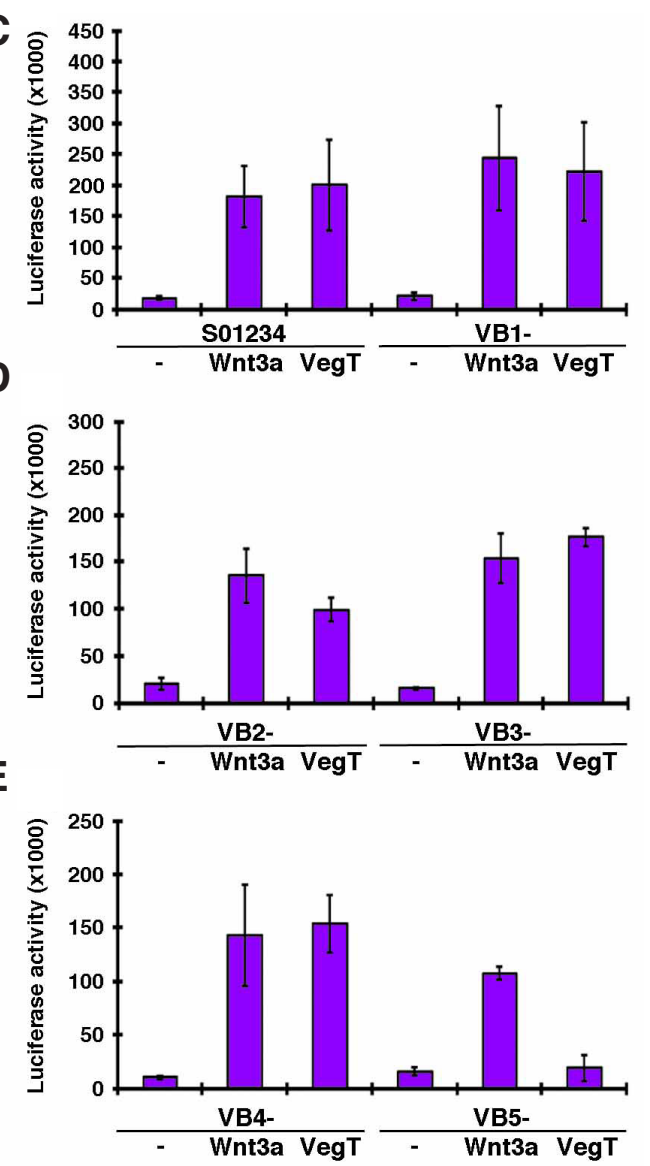

Fig. 3. VegT activates siamois promoter through T-box binding sites. (A) Nucleotide sequence of the $0.4 \mathrm{~kb}$ siamois promoter fragment. The first 12 nucleotides of the siamois coding sequence with its deduced amino acids and 402 nucleotides of siamois promoter region $5^{\prime}$ to the transcription start site (arrow) are shown. The Tcf/Lef-binding sites are indicated as SO, S1, S2, S3 and S4, as previously reported (Brannon et al., 1997). The putative T-box binding sites are boxed and identified above the sequence, as VB1, VB2, VB3, VB4 and VB5. All these sites conform to the T-box binding consensus. (B) Schematic representation of siamois reporter constructs mutated at the VegT-binding sites. (C-E) Luciferase reporter assay using ectoderm explants previously injected with wild-type or mutant siamois promoter reporter DNAs and synthetic mRNAs, as indicated. Mutation of VB1, VB2, VB3 or VB4 does not affect the response of siamois promoter to Wnt3a or VegT. Mutation of VB5 abolishes the activation of siamois promoter induced by VegT, but not by Wnt3a. Data represent the mean of triplicate experiments (error bars indicate s.d.).

(500 pg) and $\operatorname{Veg} T(200 \mathrm{pg})$ in the animal pole region and analyzed ectopic siamois expression in the ectoderm explants. Wnt3amRNA (2 pg) was also injected to compare its activity with these T domain proteins. RT-PCR analysis performed on ectoderm explants at the early gastrula stage clearly showed that Tbx6, Tbx16 and VegT, but not Xbra, similarly induced ectopic siamois expression, although this induction was less efficient than did Wnt3a (Fig. 1A). However, Wnt3a also induced Xnr3 expression as expected, but not Tbx6, Tbx16 and VegT. As previously reported, these T domain proteins also induced the expression of the mesoderm gene Wnt8 and the endoderm gene Sox $17 \alpha$, with the exception of Xbra, which only induced Wnt8 expression at the dose injected (Fig. 1A). This result indicates that different $\mathrm{T}$ domain proteins have distinct activity to induce siamois ectopic expression in ectoderm cells.

Among these $\mathrm{T}$ domain proteins, only $\mathrm{Veg} \mathrm{T}$ is maternally expressed and plays an important role in the formation of Spemann organizer (Zhang et al., 1998; Kofron et al., 1999), it is thus likely that Tbx6 and zebrafish Tbx16 only mimicked the activity of VegT. We then performed a dose-response analysis to see how VegT induces the expression of siamois and mesoderm and endoderm genes in the ectoderm explants. Injection of different amounts of VegT mRNA (1000 pg, 400 pg and 100 pg) indicated a dosedependent manner of siamois induction. VegT induced siamois expression at high and intermediate doses, but not at low dose, while it induced the expression of the endoderm gene Sox $17 \alpha$ only at high dose. The expression of the ventral mesoderm gene Wnt8 was induced both at high and low doses (Fig. 1B). These observations demonstrate that some $\mathrm{T}$ domain proteins, in particular VegT, are able to activate siamois expression.

\section{Tcf/Lef site-independent activation of siamois promoter reporter by VegT}

To further examine how VegT activates siamois expression, we first tested if and how it activates the siamois promoter reporter. A previously characterized $0.8-\mathrm{kb}$ fragment of the siamois promoter was shown to contain three typical consensus Tcf/Lef-binding sites, CTTTG $(\mathrm{A} / \mathrm{T})(\mathrm{A} / \mathrm{T})$ (S0, S1 and S3), and two additional sites (S2 and S4) differing from the Tcf/Lef core consensus sequence at the 3'-most base (Brannon et al., 1997; Fig. 2A; see also Fig. 3A). In ectoderm explants, the wild-type promoter reporter (S01234) was strongly 
activated by Wnt signalling through injection of Wnt3a mRNA (Fig. 2B). Consistent with its ability to induce siamois expression in ectoderm explants, coinjection of VegT mRNA (400 pg) with S01234 promoter reporter DNA strongly increased the luciferase activity (Fig. 2B). We then tested the activity of VegT on the S24 promoter reporter, which was mutated at the three typical consensus Tcf/ Lef-binding sites (S0: CTTTGAA to ATTCCAA, S1: ATCAAAG to ATGAAAC, S3: ATCAAAG to ATGAATTC) and did not respond to Wnt signalling (Brannon et al., 1997). Indeed, coinjection of Wnt3a mRNA (2 pg) with S24 promoter reporter DNA did not significantly increase the luciferase activity (Fig. 2B), however, coinjection of VegT mRNA (400 pg) with S24 promoter reporter DNA increased the luciferase activity similarly as its coinjection with the wild-type S01234 promoter reporter (Fig. 2B). This result clearly indicates that VegT could activate siamois promoter independently of Tcf/ Lef-binding sites.

\section{VegT activates siamois expression through T-box binding sites}

The above observations led us to examine whether consensus sequence for T-box binding site, $\operatorname{TNNCAC}(\mathrm{C} / \mathrm{T})(\mathrm{T} / \mathrm{C})$, as determined by previous works (Kispert and Hermann, 1993; Conlon et al., 2001), was present in the siamois promoter sequence. We identified 5 potential consensus sites within the $0.4 \mathrm{~kb}$ siamois promoter region upstream of the transcription start site, which we designated as VB1, VB2, VB3, VB4 and VB5 for VegT-binding sites (Fig. 3A). VB1 is located 8 nucleotides upstream of the S0 Tcf/ Lef site, while VB2 is located 14 nucleotides downstream. VB3 is located 51 nucleotides upstream of the S1 Tcf/Lef site, while VB4 is located 29 nucleotides upstream of the S3 Tcf/Lef site. VB5 is located 8 nucleotides upstream of the transcription start site (Fig. $3 \mathrm{~A})$. To analyze the contribution of each of these putative VegTbinding sites in the activation of siamois promoter by VegT, we have individually mutated the core consensus VegT-binding sequence in the wild-type S01234 promoter (Fig. 3B). The resulting mutant promoter reporters were designated as VB1 - (TTACACTT to TTAATCTT), VB2- (TCTCACTC to TCTAGATC), VB3- (TGACACCT to TGAATTCT), VB4- (TGTCACTC to TGTAGATC) and VB5- (TCTCACCT to TCTCGAGT). We then used ectoderm explants to test the activation of these mutant reporters in response to VegT, in comparison with Wnt3a. Our results showed that the siamois promoter mutants in the VB1, VB2, VB3 or VB4 site exhibited similar background activation in the ectoderm as the wild-type S01234 promoter, and that they all responded similarly to the induction by Wnt3a and VegT (Fig. 3 C-E). However, mutation of the VB5 site did not reduce the background activation but completely abolished the activation by VegT, but not by Wnt3a (Fig. 3E). These observations indicate that the VB5 site, which is in close proximity of the transcription start site, is indispensable for the activation of siamois promoter by VegT, while individual mutation of VB1 to VB4 sites has no effect. However, we cannot exclude the possibility that a combination of these sites may be required to respond to VegT.

\section{VegT binds to siamois promoter}

To provide further evidence that VegT directly regulates siamois expression, we performed ChIP experiments to examine if VegT indeed binds to the siamois promoter. One-cell stage embryos were injected in the animal pole region with Flag-tagged VegT mRNA (500 pg) and cultured to the early gastrula stage. Chromatin from both uninjected and injected embryos was immunoprecipitated
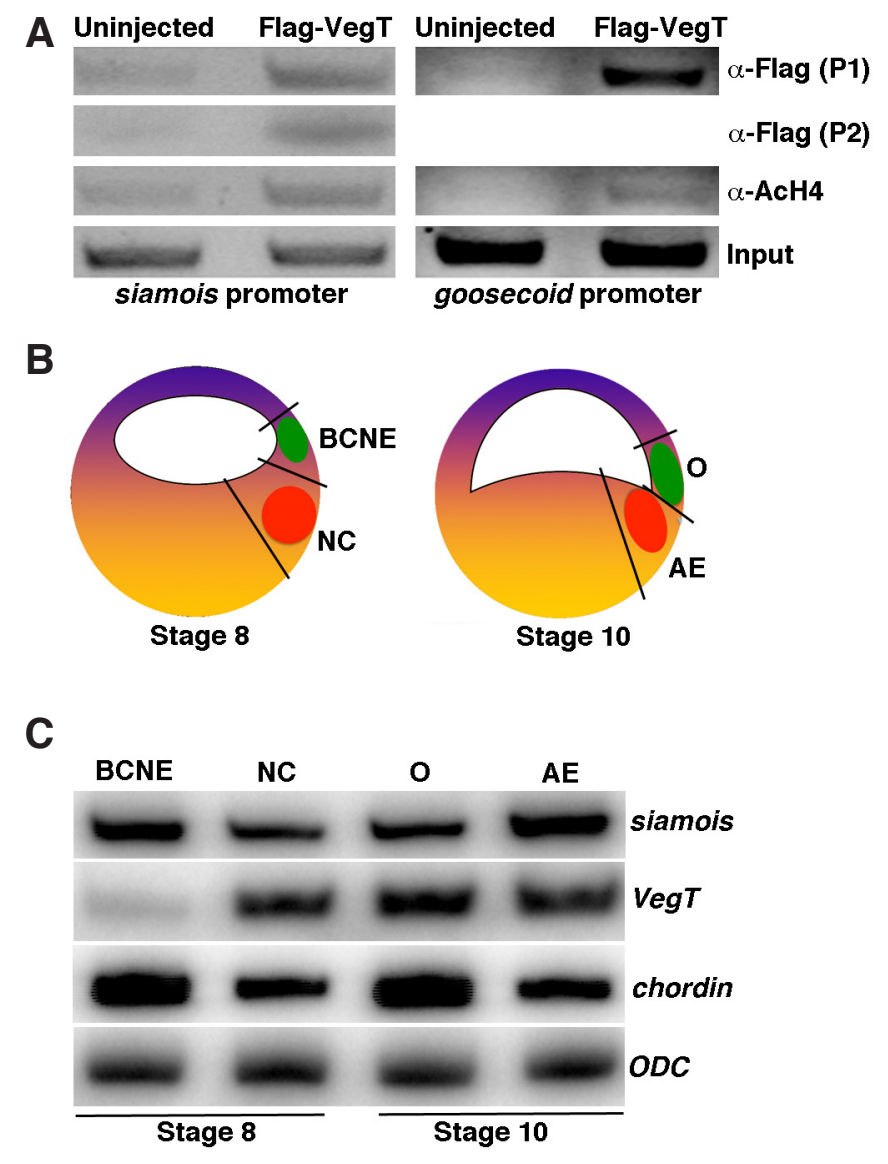

Fig. 4. Binding of VegT to the siamois promoter and overlapping expression domains between VegT and siamois at blastula and gastrula stages. (A) ChIP assays were performed using early gastrula stage embryos previously injected at the 1-cell stage with Flag-tagged VegT $m R N A$. A representative semi-quantitative $P C R$ result is shown with siamois and goosecoid promoters. $P 1$ and $P 2$ refer to siamois primer pair 1 and 2. PCR amplifications were also performed on chromatin isolated before immunoprecipitation to control the input level. (B) Schematic representation of the regions dissected at blastula and gastrula stages. (C) RT-PCR analysis of the expression of siamois, VegT and chordin in blastula chordin- and noggin-expressing (BCNE) center and Nieuwkoop center (NC) at the blastula stage, and in the Spemann organizer (O) and anterior endoderm (AE) at the early gastrula stage. ODC was a loading control.

using anti-Flag M2 antibody and analyzed by semi-quantitative PCR. The results showed that, in the presence of VegT, a -500/-168 (relative to transcription start site as +1 ) siamois promoter region containing VB1, VB2 and VB3 sites was amplified using primer pair 1 from chromatin precipitated by anti-Flag M2 antibody (Fig. 4A). A -174/+26 siamois promoter region containing VB4 and VB5 sites was also amplified using primer pair 2 (Fig. 4A). As a positive control, we have examined the goosecoid promoter, which was shown to be a direct target of VegT (Sudou et al., 2012), to validate the ChIP experiment. Consistent with previous observations, a -390/-167 goosecoid promoter region was amplified (Fig. 4A). This indicates that VegT binds to the siamois promoter, a region with also an increased acetylated histone $\mathrm{H} 4$ mark (Fig. 4A), reflecting an opened state of chromatin in this location (Shogren-Knaak et al., 2006). This result further demonstrates that siamois is a direct target of VegT. 
The expression of siamois is first detected in the dorsal animal region of the blastula chordin- and noggin-expressing (BCNE) center at the blastula stage, and then in the anterior endoderm (AE) at the early gastrula stage (Kuroda et al., 2004; Rankin et al., 2011; Sudou et al., 2012). To examine if the expression domains of siamois and VegToverlaps at blastula and gastrula stages, we dissected the regions corresponding to BCNE and Nieuwkoop center (NC) at stage 8, and the Spemann organizer and anterior endoderm at stage 10 (Fig. 4B). The expression of siamois and VegT was detected in all these regions although VegT was only weakly detected in the BCNE at stage 8 (Fig. $4 \mathrm{C}$ ). This result further supports the possibility that VegT is involved in the regulation of siamois expression.

\section{Functional interaction between VegT and Wnt signalling in the activation of siamois expression}

We have established that siamois is a direct target gene of VegT, the question remains how VegT and Wnt signalling interact to regulate siamois expression. We thus used the wild-type and different mutant siamois promoter reporters to examine this issue. First, low dose of VegTmRNA (100 pg) was coinjected with low dose of Wnt3amRNA (0.2 pg). We found that VegT strongly synergized with Wnt3a to activate the wild-type S01234 siamois promoter (Fig. $5 A)$. We then tested if VegT also cooperates with different Tcf factors (Tcf1, Tcf3 and Tcf4) to activate siamois promoter reporters. Injection of high amount of Tcf1 mRNA (200 pg) potently activated siamois promoter reporter, and injection of Tcf4 mRNA (200 pg) moderately activated siamois promoter reporter, while injection of Tcf3 mRNA (200 pg) had no effect (Fig. S2). This is consistent with a previous study showing that high dose of Tcf1 increases the expression of Wnt target genes in the ventral region of early gastrula (Standley et al., 2005). When low dose of Tcf1, Tcf3 or Tcf4 mRNA (50 pg) was coinjected with low dose of VegT mRNA (100 pg), we found that VegT cooperated with Tcf1, but not Tcf3 or Tcf4, to activate the wild-type siamois promoter reporter (Fig. 5A; Fig. S2). Unexpectedly, VegT also cooperated with Wnt3a or Tcf1 to activate the mutated S24 siamois promoter reporter lacking all consensus Tcf/Lef binding sites, which does not respond to Wnt signalling (Fig. 5B). In addition, we also observed a strong synergy between VegT and Wnt3a or Tcf1 in the activation of the VB5- siamois promoter reporter, which does not respond to VegT alone (Fig. 5C). This result indicates that VegT functionally interacts with Wnt signalling to activate siamois expression, and that the presence of either Tcf/Lef-binding sites or VegT-binding sites may be sufficient for the interaction. We further tested this possibility by deleting the VB2 to VB4 sites, and mutating the VB5 site in the S24 construct (Fig. S3A). In this case, no cooperation between VegT and Tcf1 could be observed (Fig. S3B), indicating that the presence of VB1 site alone is not sufficient to respond to VegT.

To see if the activity of VegT is required for the activation of the siamois promoter reporter (S01234) induced by Wnt signalling, we injected at the 2-cell stage in the animal pole region synthetic mRNA (200 pg) corresponding to the dominant negative VegT mutant (VegT-ENR), in which the DNA binding domain of VegT (amino acids 1 to 284) is fused the Drosophila Engrailed repressor domain (amino acids 2 to 298), with Wnt3a mRNA (2 pg) or Tcf1 mRNA (200 pg), and assayed luciferase activity in ectoderm explants at the early gastrula stage. The result showed that blocking the activity of VegT strongly inhibited the activation of the siamois promoter induced by Wnt3a or Tcf1 (Fig. 5D). This result further suggests that VegT cooperates with Wnt signalling and is required for siamois expression. We then performed RT-PCR and in situ hybridization analyses to confirm the functional interaction between VegT and Wnt signalling. As observed in siamois promoter reporter assays, coinjection of low amounts of VegT and Wnt3a mRNAs was able to strongly induce siamois expression in ectoderm explants (Fig. $6 \mathrm{~A}$ ), indicating a synergy of the two pathways. In addition, the ectopic siamois expression induced by Wnt3a or Tcf1 in ectoderm explants was strongly inhibited by the presence of VegT-ENR (Fig. 6A). Conversely, inhibition of Wnt signalling by the dominant negative Tcf3 (dnTcf3), with the N-terminal 87 amino acids of the $\beta$-catenin-binding domain deleted, also potently inhibited siamois ectopic expression induced by VegT (Fig. S4). This result clearly suggests that VegT and Wnt signalling are mutually required for siamois expression.

In the whole embryo, to examine the requirement of both VegT
Fig. 5. VegT synergizes with Wnt signalling to activate siamois promoter reporter. Two-cell stage embryos were injected with synthetic mRNA along with wild-type or different mutant siamois promoter DNAs, as indicated, in the animal pole region and ectoderm explants were dissected at the early gastrula stage for luciferase assay. (A) At low dose, VegT synergizes with Wnt3a or Tcf1 to activate luciferase activity driven by the $\mathbf{S 0 1 2 3 4}$ wild-type siamois promoter. (B) A similar synergy can be observed using the S24 mutant promoter lacking the three canonical Tcf/Lef-binding sites. (C) VegT cooperates with Wnt3a or Tcf1 to activate the VB5-siamois promoter reporter mutated at VB5 site. (D) The dominant negative VegT mutant blocks activation of the S01234 wildtype siamois promoter reporter induced by injection of high dose of Wnt3a or Tcf1 $m R N A$. Data represent the mean of triplicate experiments (error bars indicate s.d.).
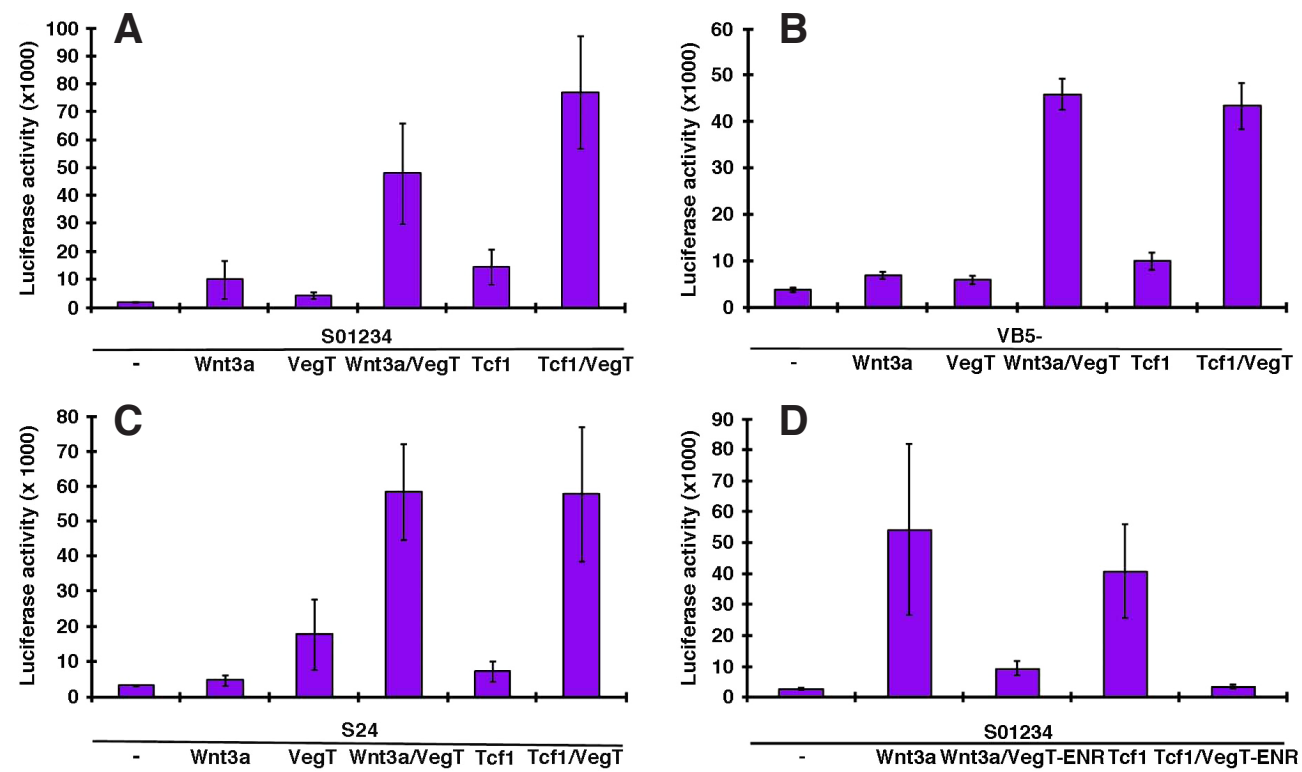
and Wnt signalling in the formation of Spemann organizer, we injected VegT-ENR mRNA (50 pg), dnTcf3 mRNA (50 pg) or the same amounts of both VegT-ENR and dnTcf3 mRNAs in the dorsal equatorial region at the 4-cell stage, in situ hybridization was first performed to analyze the expression of chordin at the early gastrula stage. Single injection of $d n T c f 3$ or VegT-ENR inhibited chordin expression in the dorsal region (Fig. 6 B-D). Coinjection of $d n T c f 3$ and VegT-ENR nearly blocked chordin expression (Fig. $6 \mathrm{E})$. This result was further confirmed by RT-PCR analysis, which also showed an increase in the expression level of the ventral mesoderm gene BMP4 in singly or doubly injected early gastrula (Fig. S5). Consistent with these molecular analyses, examination of the phenotypes at the late tail-bud stage indicates that single injection of VegT-ENR or dnTcf3 resulted in head deficiency and shortened trunk and tail regions (Fig. $6 \mathrm{~F}-\mathrm{H}, \mathrm{J}$ ). Coinjection of VegT-ENR and $d n T c f 3$ resulted in the absence of head and severely affected trunk and posterior regions (Fig. 6 I,J). These observations sug-

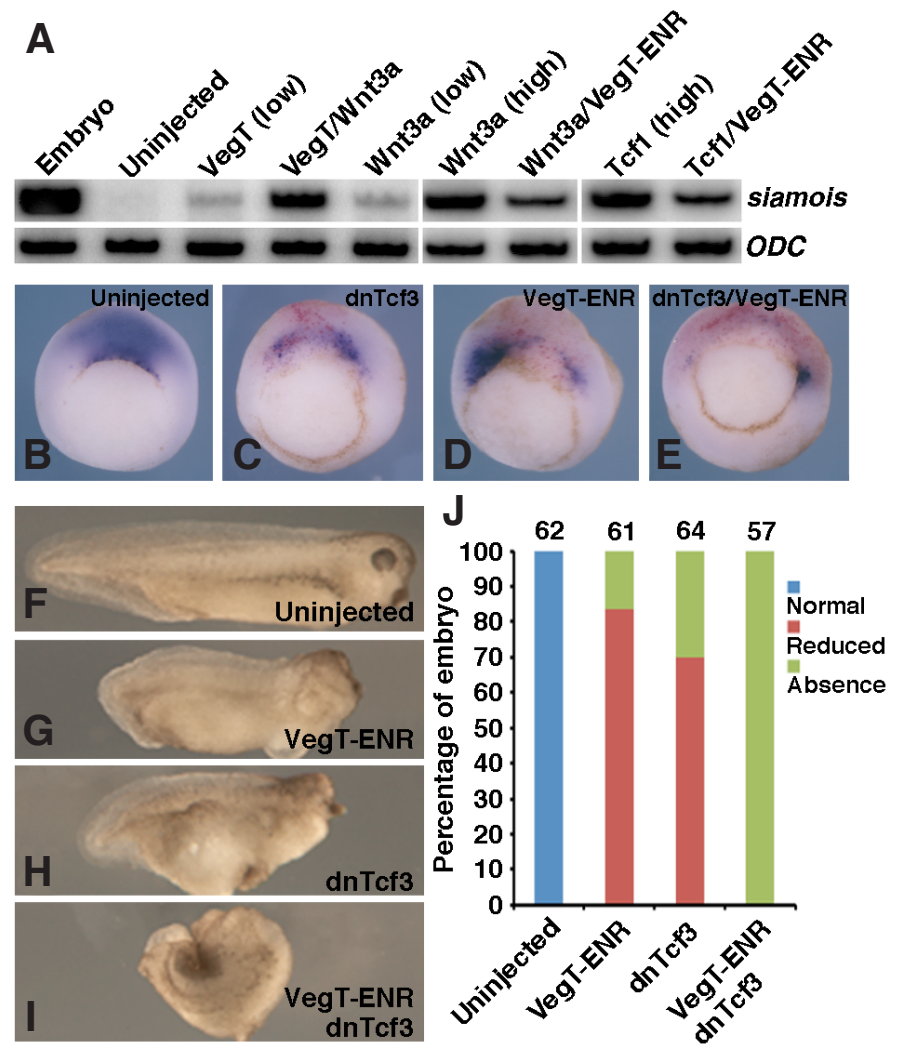

Fig. 6. Functional interaction between VegT and Wnt signalling in regulating Spemann organizer formation. (A) RT-PCR analysis of siamois ectopic expression in ectoderm explants injected with indicated mRNAs. VegT synergizes with Wnt signalling to induce siamois expression. It is also required for siamois expression activated by Wnt signalling. ODC was a loading control. (B-E) In situ hybridization analysis of chordin expression in control(B) and stage 10.5 early gastrula previously injected with dnTcf3 (C) or VegT-ENR (D) mRNA, or coinjected with dnTcf3 and VegT-ENR mRNAs (E). $\beta$-gal staining was used to localise the injected region. Coinjection of dnTcf3 and VegT-ENR more efficiently blocks chordin expression than single injection of dnTcf3 or VegT-ENR. (F-I) Phenotypic analysis of control (F) and embryos injected with VegT-ENR (G), dnTcf3 (H) or dnTcf3 and VegT-ENR (I), at the late tail-bud stage. (J) Statistical analysis of the phenotypes. Numbers on the top of each stacked column indicate total embryos scored from two independent experiments. gest that maternal Wnt signalling and VegT cooperate to regulate the formation of Spemann organizer and anterior development by activating the expression of siamois. In addition, when RTPCR analysis was performed at the start of zygotic transcription (stage 8) on whole embryos injected with VegT-ENR or dnTcf3, or coinjected with VegT-ENR and dnTcf3, the result indicates that blockade of maternal Wnt signalling and VegT function inhibited siamois expression (Fig. S6), indicating that thay are required for initiation of siamois expression.

VegT cooperates with Wnt signalling to induce the expression of Xnr genes (Agius et al., 2000). To examine which $X n r$ genes are directly regulated VegT and Wnt signalling, we injected VegT $(100 \mathrm{pg})$ and Wnt8 (1 pg) mRNAs in the animal pole region at the 2-cell stage, without or with cer-S mRNA (200 pg) to inhibit Nodal signalling (Piccolo et al., 1999). PCR analysis on ectoderm explants dissected at the early gastrula stage showed that Cerberus-short inhibited the expression of Xnr2, Xnr4 and Xnr6, but had no effect on the expression of $X n r 1$ and $X n r 3$, induced by VegT and Wnt8 (Fig. S7). This result is consistent with previous studies showing that $X n r 1$ is a direct target of VegT and Wnt signalling (Kofron et al., 1999; Hyde and Old, 2000), and that Xnr3 is a direct target of Wnt signalling (McKendry et al., 1997). Thus, both VegT and Wnt signalling should cooperate in the formation of the Spemann organizer.

\section{Discussion}

In this study, we showed that VegT binds to the siamois promoter and directly activates siamois expression through putative VegTbinding sites. In addition, it also cooperates with Wnt signalling to regulate siamois expression and the formation of Spemann organizer in the Xenopus embryo. Our finding suggests that the presence of either Tcf/Lef-binding sites or VegT-binding sites is sufficient for a synergistic interaction between VegT and Wnt signalling.

\section{Direct regulation of siamois by VegT}

Veg $T$ is a maternal mRNAlocalized vegetally in the early embryos, it is also transcribed zygotically within the equatorial region of the early gastrula (Zhang and King, 1996; Lustig et al., 1996; Stennard et al., 1996; Horb and Thomsen, 1997). Maternal VegT has been shown to play an essential role in embryonic axis formation (Zhang et al., 1998; Xanthos et al., 2002). The homeobox gene siamois is zygotically expressed in the dorsal region including the Spemann organizer and the prospective anterior endoderm (Lemaire et al., 1995; Kuroda et al., 2004; Rankin et al., 2011; Sudou et al., 2012), and is required for the formation of dorsal axis (Kessler, 1997; Fan and Sokol, 1997). In addition, we showed that the expression domains of VegT and siamois overlap at the blastula and early gastrula stages. This supports the possibility that VegT may be involved in the regulation of siamois expression. Indeed, we have demonstrated that a subset of $T$ domain transcription factors could induce siamois ectopic expression in ectoderm cells. In addition, VegT could also activate the siamois promoter independently of Tcf/Lef-binding sites.

The consensus sequence TNNCAC $(\mathrm{C} / \mathrm{T})(\mathrm{T} / \mathrm{C})$ has been shown to be the potential binding site for VegT (Kispert and Hermann, 1993; Conlon et al., 2001), according to this criterion, we have identified 5 putative VegT-binding sites within the $0.4 \mathrm{~kb}$ siamois promoter region. Individual mutation of any one of the four distal sites (VB1 
to VB4) did not affect the response of the siamois promoter reporter to VegT. By contrast, mutation of the proximal VB5 site located at close proximity of the transcription start site indicates that it is indispensable for the activation of siamois promoter by VegT, but not by Wnt signalling. However, this result does not allow us to conclude whether VB5 site is sufficient for VegT-induced siamois transcription, or whether a combination of other sites may be also necessary for binding of VegT to the siamois promoter. It remains possible that the presence of VB5 site alone may not be sufficient and that the presence of multiple sites may be required for VegT to activate siamois transcription. Consistent with this proposal, our ChIP assay showed that VegT was able to bind to the regions including VB1, VB2 and VB3 sites, as well as VB4 and VB5 sites.

We have also observed that different $T$ domain proteins exhibit distinct activity to activate siamois expression although they were shown to bind essentially to the same core consensus sequence (Kispert and Hermann, 1993; Conlon et al., 2001). In particular, VegT, but not Xbra, activates siamois ectopic expression in ectoderm cells. There are at least two possibilities, which could explain this difference. First, Xbra usually binds to palindromic sequence, with two core sequences and without intervening nucleotides, which is not the case in the siamois promoter. However, VegT can interact with a single core sequence (Conlon et al., 2001). Second, the functional difference between Xbra and VegT may depend on their $\mathrm{T}$ domains. There is evidence showing that the lysine residue $(\mathrm{K})$ at position 149 of $\mathrm{Xbra}$ and the asparagine residue $(\mathrm{N})$ at the corresponding position of VegT may play a differential role in the inductive activity for Xbra and VegT (Conlon et al., 2001). In addition, the specificity of $\mathrm{T}$ domain proteins could be conferred by the $\mathrm{N}$-terminal region, which interacts with different protein partners to elicit a specific response (Messenger et al., 2005). Thus, these differences may largely account for their differential activity to induce siamois expression.

\section{Interaction between VegT and Wnt signalling}

Another finding made in this work concerns the mode of interaction between Wnt signalling and VegT in the activation of the siamois promoter. Since both Tcf/Lef-binding sites and VegT-binding sites are present in the siamois promoter, it can be expected that the two pathways functionally interact to regulate the transcription of siamois. We found that, in reporter assays, they indeed functionally interact and act synergistically to activate siamois promoter reporter. However, it is surprising that VegT and Wnt signalling also synergize to activate mutant promoters lacking either all consensus Tcf/Lefbinding sites or the VB5 site, which is required for VegT-induced siamois transcription in the absence of Wnt signalling. Promoter deletion analysis indicated that the absence of all the consensus Tcf/ Lef-binding sites and the four distal VegT-binding sites completely abolished the activation of siamois promoter reporter in the dorsal region of the early gastrula (Brannon et al., 1997). Consistent with this observation, our in vitro analysis indicated that removal of the all the consensus Tcf/Lef-binding sites and deletion or mutation of VB2 to VB5 sites abolished the interaction between VegT and Tcf1 to activate siamois promoter in ectoderm cells. Further analysis through simultaneous mutation of all VegT-binding sites should help to clarify their implication in VegT response of siamois promoter.

The functional interaction between VegT and Wnt signalling raises the possibility that the two pathways may physically interact, and this is supported by a previous study showing that VegT binds directly to Tcf3 (Cao et al., 2007). A physical interaction between VegT and T cell factors could explain why dominant negative VegT mutant blocks siamois expression induced by Wnt signalling, and vice versa. Nevertheless, our reporter assay using the wild-type siamois promoter did not reveal a functional interaction between VegT and Tcf3 or Tcf4. We also failed to detect a direct interaction between VegT and Tcf1 by coimmunoprecipitation (data not shown). Thus, it is still unclear how VegT and Tcf1 synergize to activate siamois expression. It remains possible that there is weak and/or indirect interaction between VegT and Tcf1, this could be sufficient for their functional cooperation on the siamois regulatory region.

The implication of VegT in the formation of Spemann organizer has been well documented. Depletion of maternal VegT strongly reduces the transcription of a panel genes expressed in the Spemann organizer, including siamois. Simultaneous depletion of VegT and $\beta$-catenin further reduces siamois expression before and during gastrulation (Xanthos et al., 2002). This suggests that VegT has the potential to regulate siamois expression. Our present study thus provides a molecular mechanism underlying the regulation of siamois by VegT and Wnt signalling in the formation of Spemann organizer. It also helps to understand the mode of interaction between $\mathrm{T}$ domain proteins and Wnt signalling.

\section{Materials and Methods}

\section{Embryos and ectoderm explants}

Xenopuseggs were obtained from females injected with $500 \mathrm{IU}$ of human chorionic gonadotropin (Sigma) and artificially fertilized. Eggs were dejellied with $2 \%$ cysteine hydrochloride $(\mathrm{pH} 7.8)$ and kept in $0.1 \times$ modified Barth solution (MBS) to appropriate stages. Microinjections of embryos were done in $0.1 \times$ MBS containing 3\% Ficoll-400. Dissection of ectoderm explants was performed in $1 \times$ MBS and cultured in this solution until appropriate stages. The identification of Tbx6 target genes using hormone-inducible version of Tbx6VP16 was performed as previous described (Li et al., 2006). LacZ mRNA (500 pg) was injected as a cell lineage tracer and $\beta$-galactosidase ( $\beta$-gal) staining was performed using red-gal as a substrate (Li et al., 2010).

\section{Plasmid constructs and site-directed mutagenesis of the siamois promoter}

The siamois reporter constructs (S01234 and S24), Xenopus Wnt3a, Xbra, Tbx6, Tbx6VP16-GR, Flag-tagged VegT (Flag-VegT), dominant negative VegT (VegT-ENR), dominant negative Tcf3(dnTcf3) and Cerberusshort (cer-S) were described previously (Brannon et al., 1997; Piccolo et al., 1999; Shi et al., 2002; Li et al., 2006). The full-length zebrafish Tbx16 coding sequence was PCR-amplified from embryonic mRNA according to the sequence in the database (accession number NM_131058) and cloned into pCS2 vector. Myc-tagged Xenopus Tcf1, Tcf3 and Tcf4 coding sequences were cloned into pCS2MT vector inframe with the six myc epitopes. Mutations of the core consensus VegT-binding (VB) sites in the siamois promoter (S01234) were performed using QickChange XL sitedirected mutagenesis kit (Stratagene) according to manufacture's instructions. The mismatched oligonucleotides used to mutate each VB site are as follow: VB1, 5'-GAATAAATAATACAAGCTTAAGTAAGGGCTTTG-3'; VB2, 5'- CTTGCCAACTTCTCTAGATCAGTCCTTCA-3'; VB3, 5'-GCTGCCAAGTTAGAATTCATATGGGACAG-3'; VB4, 5'- GCATATGTATAAAACATCTAGACTGACCAATAACC-3'; VB5, 5'- CTCCCAGGACAGAGCTCACCAACATTTTGG-3'. Mutated nucleotides in the core consensus VB sites are underlined. The S4VB1 siamois reporter construct was obtained by deletion in the $\mathrm{S} 24$ construct of an internal Eco RI fragment comprising the 3 consensus Tcf/Lef-binding sites and VB2 to VB4 sites, followed by mutation of the VB5 site. All constructs were sequenced before use. 


\section{Luciferase assay}

The reporter plasmids (200 pg) were either injected alone or coinjected with different synthetic mRNAs in the animal pole region at the 2-cell stage. Cell lysates were prepared from 10 ectoderm explants dissected at the early gastrula stage and luciferase assay was performed using the luciferase assay system (Promega). The luciferase activity was measured using a Lumat LB 9507 luminometer (Berthold). The experiments were carried out in triplicate using different batches of embryos and the data were analyzed using Student's $t$-test.

\section{$R T-P C R$ and in situ hybridization}

Extraction of total RNA from whole embryos or ectoderm explants was performed using guanidine isothiocyanate/phenol followed by $\mathrm{LiCl}$ precipitation to remove genomic DNA and polysaccharides. RNA samples were further treated with RNAse-free DNAse I (Roche) and were reversetranscribed using 200 units M-MLV reverse transcriptase (Invitrogen). PCR primers for siamois, goosecoid, chordin, BMP4, Wnt8, Sox17a Xnr1, Xnr2, Xnr3, Xnr4, Xnr6 and ODC (ornithine decarboxylase) were as described previously (Umbhauer et al., 2000; Shi et al., 2002; Li et al., 2006, 2013). One-twentieth of the reverse-transcribed cDNA was used for PCR amplification in a reaction mixture containing $1 \mu \mathrm{Ci}$ of $\left[\alpha-{ }^{32} \mathrm{P}\right] \mathrm{dCTP}$ (GE Healthcare). PCR products were resolved on a $5 \%$ non-denaturing polyacrylamide gel and visualized by a Phospho-Imager (BioRad). For some experiments, unlabelled PCR products were resolved on a $1.5 \%$ agarose gel. Wholemount in situ hybridization was performed according to standard protocol (Harland, 1991). The probe was labelled using digoxigenin-11-UTP and appropriate RNA polymerase (Roche).

\section{Chromatin immunoprecipitation (ChIP)}

ChIP assay was performed as described (Li et al., 2013), with minor modifications. One-cell stage embryos were injected in the animal pole region with 500 pg Flag-tagged VegT mRNA, 20 uninjected or injected embryos at the early gastrula stage were homogenized in $1 \mathrm{ml}$ lysis buffer (2.2 M sucrose, $3 \mathrm{mM} \mathrm{CaCl}, 10 \mathrm{mM}$ Tris- $\mathrm{HCl}, \mathrm{pH} 7.5,0.5 \%$ Triton X-100, 1 $\mathrm{mM}$ PMSF, $1 \mu \mathrm{g} / \mathrm{ml}$ aprotinin, $1 \mu \mathrm{g} / \mathrm{ml}$ pepstatin) and centrifuged at 53,000 $\mathrm{rpm}$ for 3 hours at $4^{\circ} \mathrm{C}$ using a TL-100 ultracentrifuge with a fixed-angle rotor (Beckman). The nuclei pellet was resuspended in $360 \mu \mathrm{l}$ of nuclear isolation buffer $(0.25 \mathrm{M}$ sucrose, $10 \mathrm{mM}$ Tris- $\mathrm{HCl}, \mathrm{pH} 7.5,3 \mathrm{mM} \mathrm{CaCl}, 1$ $\mathrm{mM} \mathrm{PMSF}, 1 \mu \mathrm{g} / \mathrm{ml}$ aprotinin, $1 \mu \mathrm{g} / \mathrm{ml}$ pepstatin). Proteins were crosslinked to DNA by adding formaldehyde (37\%) directly to the nuclear suspension to a final concentration of $1 \%$ and incubated for 10 minutes on ice and then 20 minutes at room temperature. The lysate was sonicated 16 times by using a sonicator set to $20 \%$ low amplitude to reduce DNA length between 200 to $800 \mathrm{bp}$. Sonicated chromatin $(25 \mu \mathrm{g})$ was incubated with 10 $\mu \mathrm{g}$ of anti-Flag M2 antibody (Sigma) or $8 \mu \mathrm{g}$ of anti-acetylated histone $\mathrm{H} 4$ $(\mathrm{AcH} 4)$ antibody (Millipore). ChIP products were analyzed by PCR using specific primers for siamois and goosecoid promoters (siamois primer pair 1: 5'-CATCCTTGGCCTGTTCTAAG-3' and 5'-GGCAAGGTCAAAGATGTGAT-3'; siamois primer pair 2: 5'-CCTTGCCAATTCTTTCAGGG-3' and 5'-CCTCATAGGTCATCTCTGTC-3'; goosecoid: 5'-CCAGAGAAACAAAACAGTCATTCC-3' and 5'-GCAGACTCTCCCTGTAGTTATTCAC-3'). The experiments were performed in triplicate.

\section{Acknowledgements}

We would to thank Dr D. Kimelman, J.C. Smith, E.M. De Robertis for plasmid constructs used in this study, and members of the laboratory for discussion and assistance in ChIP experiments. This work was supported by grants from Association Française contre les Myopathies (AFM), Ligue Nationale Contre le Cancer ( $L N C C$ ) and Agence Nationale de la Recherche (ANR) [ANR-09-BLAN-0262-03].

\section{References}

AGIUS E, OELGESCHLAGER M, WESSELY O, KEMP C, DE ROBERTIS E M (2000). Endodermal Nodal-related signals and mesoderm induction in Xenopus.
Development 127: 1173-1183.

BRANNON M, GOMPERTS M, SUMOY L, MOON R T, KIMELMAN D (1997). A beta-catenin/XTcf-3 complex binds to the siamois promoter to regulate dorsal axis specification in Xenopus. Genes Dev 11: 2359-2370.

CARNAC G, KODJABACHIAN L, GURDON J B, LEMAIRE P (1996). The homeobox gene Siamois is a target of the Wnt dorsalisation pathway and triggers organiser activity in the absence of mesoderm. Development 122: 3055-3065.

CAO Y, SIEGEL D, DONOW C, KNÖCHEL S, YUAN L, KNÖCHEL W (2007). POU-V factors antagonize maternal VegT activity and beta-Catenin signaling in Xenopus embryos. EMBO J 26: 2942-2954.

CASEYE S, TADAM, FAIRCLOUGH L, WYLIE C C, HEASMAN J, SMITH J C (1999). Bix 4 is activated directly by VegT and mediates endoderm formation in Xenopus development. Development 126: 4193-200.

CONLON F L, FAIRCLOUGH L, PRICE B M, CASEY E S, SMITH J C (2001). Determinants of T box protein specificity. Development 128: 3749-3758.

FAN M J, SOKOL S Y (1997). A role for Siamois in Spemann organizer formation. Development 124: 2581-2589.

FAN M J, GRÜNING W, WALZG, SOKOLSY (1998). Wnt signaling and transcriptional control of Siamois in Xenopus embryos. Proc Natl Acad Sci USA 95: 5626-5631.

HARLAND R M (1991). In situ hybridization: an improved whole mount method for Xenopus embryos. Methods Cell Biol 36: 685-695.

HEASMAN J (1997). Patterning the Xenopus blastula. Development 124:4179-4191.

HORB M E, THOMSEN G H (1997). A vegetally localized T-box transcription factor in Xenopus eggs specifies mesoderm and endoderm and is essential for embryonic mesoderm formation. Development 124: 1689-1698.

HYDE C E, OLD R W (2000). Regulation of the early expression of the Xenopus nodal-related 1 gene, Xnr1. Development 127: 1221-1229.

KESSLER D S. (1997) Siamois is required for formation of Spemann's organizer. Proc Natl Acad Sci USA 94: 13017-13022.

KISPERT A, HERMANN B G (1993). The Brachyury gene encodes a novel DNA binding protein. $E M B O \mathrm{~J}$ 12: 321-3220.

KOFRON M, DEMEL T, XANTHOS J, LOHR J, SUN B, SIVE H, OSADA S, WRIGHT C, WYLIE C, HEASMAN J (1999). Mesoderm induction in Xenopus is a zygotic event regulated by maternal VegT via TGFbeta growth factors. Development 126: 5759-5770.

KURODA H, WESSELY O, DE ROBERTIS E M (2004). Neural induction in Xenopus: requirement for ectodermal and endomesodermal signals via Chordin, Noggin, beta-Catenin, and Cerberus. PLoS Biol 2: E92.

LEMAIRE P, GARRETT N, GURDON J B (1995). Expression cloning of Siamois, a Xenopus homeobox gene expressed in dorsal-vegetal cells of blastulae and able to induce a complete secondary axis. Cell 81: 85-94.

LI H Y, BOURDELAS A, CARRON C, GOMEZ C, BOUCAUT J C, SHI D L (2006). FGF8, Wnt8 and Myf5 are target genes of Tbx6 during anteroposterior specification in Xenopus embryo. Dev Biol 290: 470-481.

LI H Y, BOURDELAS A, CARRON C, SHI D L (2010). The RNA-binding protein Seb4 is a target of MyoD and is required for myogenesis during Xenopus early development. Mech Dev 127: 281-291.

LI H Y, GRIFONE R, SAQUET A, CARRON C, SHI D L (2013). The Xenopus homologue of Down syndrome critical region protein 6 drives dorsoanterior gene expression and embryonic axis formation by antagonising polycomb group proteins. Development 140: 4903-4913.

LUSTIG K D, KROLL K L, SUN E E, KIRSCHNER M W (1996). Expression cloning of a Xenopus T-related gene (Xombi) involved in mesodermal patterning and blastopore lip formation. Development 122: 4001-4012.

MCKENDRY R, HSU S C, HARLAND R M, GROSSCHEDL R (1997). LEF-1/TCF proteins mediate wnt-inducible transcription from the Xenopus nodal-related 3 promoter. Dev Biol 192: 420-431.

MESSENGER N J, KABITSCHKE C, ANDREWS R, GRIMMER D, NUNEZ MIGUEL R, BLUNDELL T L, SMITH J C, WARDLE F C (2005). Functional specificity of the Xenopus T-domain protein Brachyury is conferred by its ability to interact with Smad1. Dev Cell 8: 599-610.

PICCOLOS, AGIUSE, LEYNSL, BHATTACHARYYAS, GRUNZH, BOUWMEESTER T, DE ROBERTIS E M (1999). The head inducer Cerberus is a multifunctional antagonist of Nodal, BMP and Wnt signals. Nature 397: 707-710.

RANKIN S A, KORMISH J, KOFRON M, JEGGA A, ZORN A M (2011). A gene 
regulatory network controlling hhex transcription in the anterior endoderm of the organizer. Dev Biol 351: 297-310.

SHI D L, BOURDELAS A, UMBHAUER M, BOUCAUT J C (2002). Zygotic Wnt/ beta-catenin signaling preferentially regulates the expression of Myf5 gene in the mesoderm of Xenopus. Dev Biol 245: 124-135.

SHOGREN-KNAAK M, ISHII H, SUN J M, PAZIN M J, DAVIE J R, PETERSON C $\mathrm{L}$ (2006). Histone H4-K16 acetylation controls chromatin structure and protein interactions. Science 311: 844-847.

STANDLEY H J, DESTRÉE O, KOFRON M, WYLIE C, HEASMAN J (2005). Maternal XTcf1 and XTcf4 have distinct roles in regulating Wnt target genes. Dev Biol 289: 318-328.

STENNARD F, CARNAC G, GURDON J B (1996). The Xenopus T-box gene, Antipodean, encodes a vegetally localised maternal mRNA and can trigger mesoderm formation. Development 122: 4179-4188.

SUDOU N, YAMAMOTO S, OGINO H, TAIRA M (2012). Dynamic in vivo binding of transcription factors to cis-regulatory modules of cer and gsc in the stepwise formation of the Spemann-Mangold organizer. Development 139: 1651-1661.

TADA M, CASEY E S, FAIRCLOUGH L, SMITH J C (1998). Bix1, a direct target of Xenopus T-box genes, causes formation of ventral mesoderm and endoderm. Development 125: 3997-4006.
TAVERNER N V, KOFRON M, SHIN Y, KABITSCHKE C, GILCHRIST M J, WYLIE C, CHO K W, HEASMAN J, SMITH J C (2005). Microarray-based identification of VegT targets in Xenopus. Mech Dev 122: 333-354.

UMBHAUER M, DJIANE A, GOISSET C, PENZO-MENDEZ A, RIOU J F, BOUCAUT J C, SHI D L (2000). The C-terminal cytoplasmic Lys-Thr-X-X-X-Trp motif in frizzled receptors mediates Wnt/beta-catenin signalling. EMBO J 19: 4944-4954.

WHITE J A, HEASMAN J (2008). Maternal control of pattern formation in Xenopus laevis. J Exp Zool B Mol Dev Evol 310: 73-84.

XANTHOS J B, KOFRON M, WYLIE C, HEASMAN J (2001). Maternal VegT is the initiator of a molecular network specifying endoderm in Xenopus laevis. Development 128: 167-180.

XANTHOS J B, KOFRON, M, TAO Q, SCHAIBLE K, WYLIE C, HEASMAN J (2002). The roles of three signaling pathways in the formation and function of the Spemann Organizer. Development 129: 4027-4043.

ZHANG J, KING M L (1996). Xenopus VegT RNA is localized to the vegetal cortex during oogenesis and encodes a novel T-box transcription factor involved in mesodermal patterning. Development 122: 4119-4129.

ZHANG J, HOUSTON D W, KING M L, PAYNE C, WYLIE C, HEASMAN J (1998). The role of maternal VegT in establishing the primary germ layers in Xenopus embryos. Cell 94: 515-524. 


\section{Further Related Reading, published previously in the Int. J. Dev. Biol.}

Zygotic expression of Exostosin1 (Ext1) is required for BMP signaling and establishment of dorsal-ventral pattern in Xenopus Yi-Er Shieh, Dan E. Wells and Amy K. Sater

Int. J. Dev. Biol. (2014) 58: 27-34

http://dx.doi.org/10.1387/ijdb.130257as

\section{Lef1 plays a role in patterning the mesoderm and ectoderm in Xenopus tropicalis}

Giulietta Roël, Yoony Y.J. Gent, Josi Peterson-Maduro, Fons J. Verbeek and Olivier Destrée Int. J. Dev. Biol. (2009) 53: 81-89

http://dx.doi.org/10.1387/ijdb.072395gr

Generating asymmetries in the early vertebrate embryo: the role of the Cerberus-like family José António Belo, Ana C. Silva, Ana-Cristina Borges, Mário Filipe, Margaret Bento, Lisa Gonçalves, Marta Vitorino, Ana-Marisa Salgueiro, Vera Texeira, Ana T. Tavares and Sara Marques Int. J. Dev. Biol. (2009) 53: 1399-1407

http://dx.doi.org/10.1387/ijdb.072297jb

Multiple interactions between maternally-activated signalling pathways control Xenopus nodal-related genes

Maria Rex, Emma Hilton and Robert Old

Int. J. Dev. Biol. (2002) 46: 217-226

http://dx.doi.org/10.1387/ijdb.11934150

Siamois cooperates with TGFbeta signals to induce the complete function of the Spemann-Mangold organizer

M J Engleka and D S Kessler

Int. J. Dev. Biol. (2001) 45: 241-250

http://dx.doi.org/10.1387/ijdb.11291853

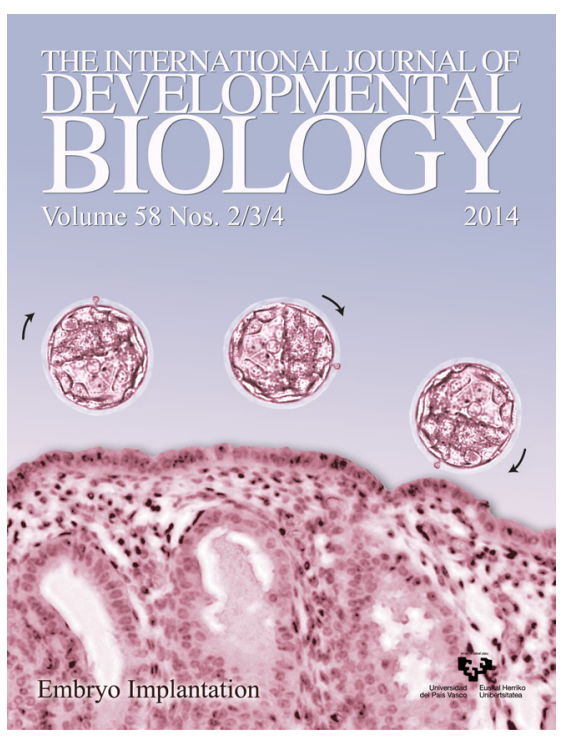

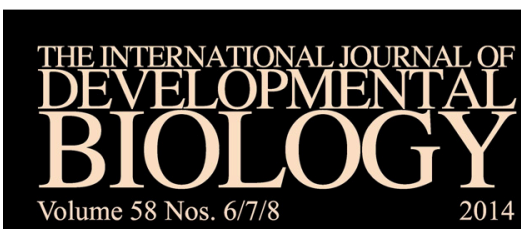
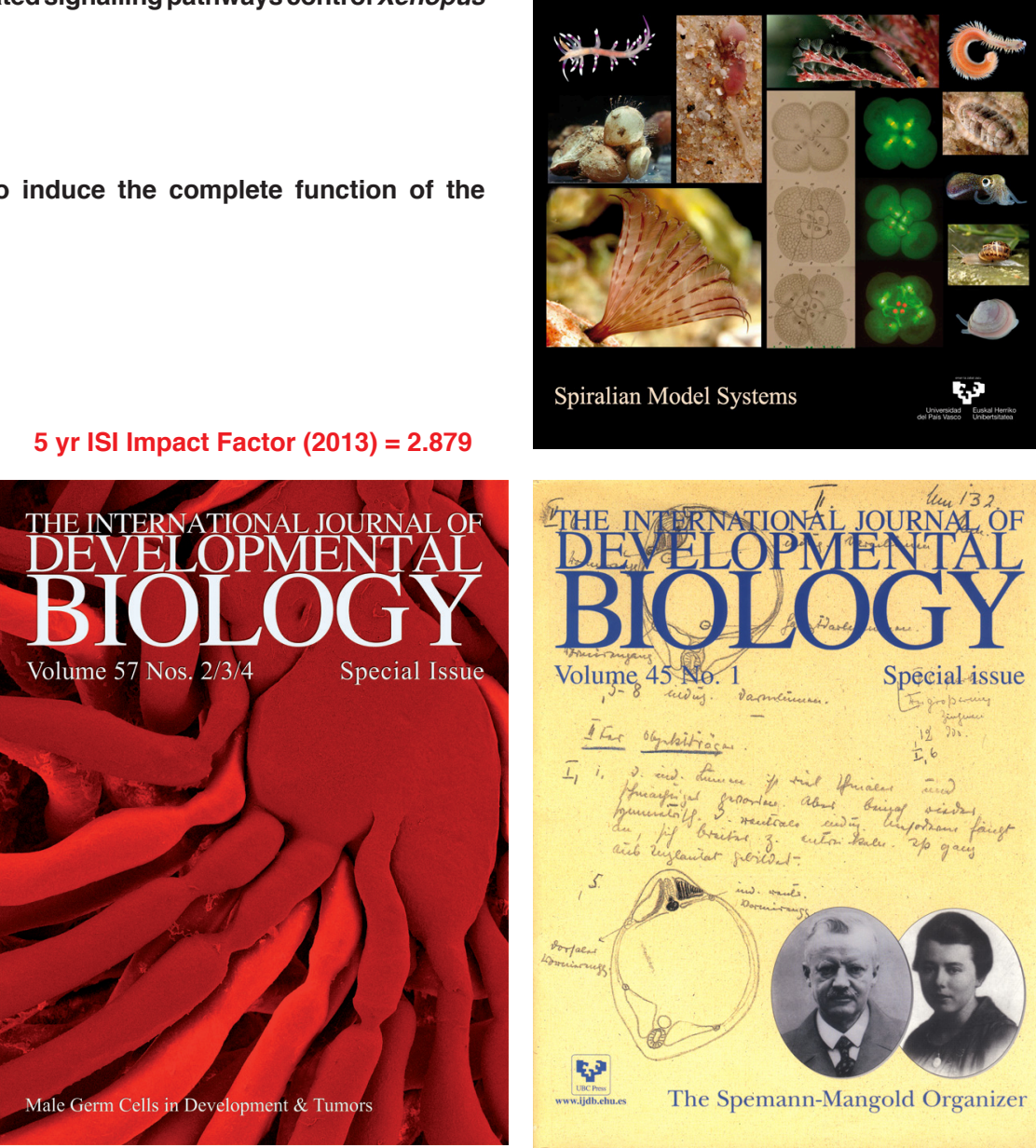

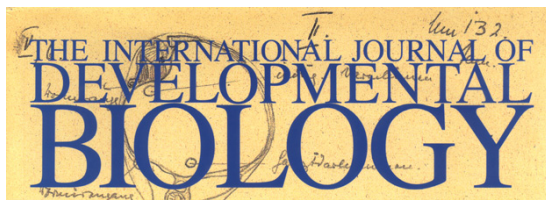

Volume 45 No. 1

Spécial issue

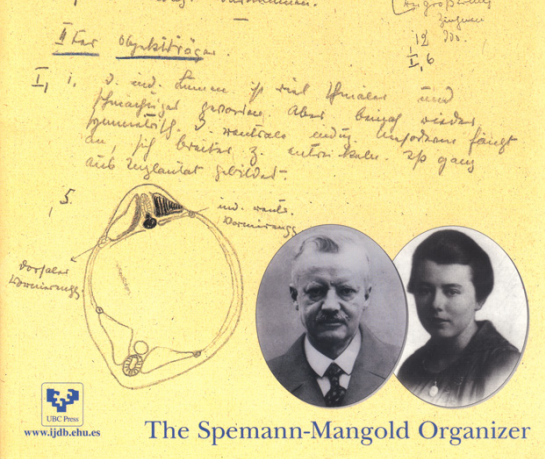

